\title{
Mind-Body Health Benefits of Traditional Chinese Qigong on Women: A Systematic Review of Randomized Controlled Trials
}

\author{
Kin-Chung Wilson Leung $\mathbb{D}^{1},{ }^{1}$ Yi-Jian Yang $\mathbb{D}^{1,2}$ Stanley Sai-Chuen Hui $\mathbb{D}^{1}{ }^{1}$ \\ and Jean Woo $\mathbb{D i D}^{2,3}$ \\ ${ }^{1}$ Department of Sports Science and Physical Education, Faculty of Education, The Chinese University of Hong Kong, \\ Hong Kong, China \\ ${ }^{2}$ CUHK Jockey Club Institute of Ageing, The Chinese University of Hong Kong, Hong Kong, China \\ ${ }^{3}$ Department of Medicine and Therapeutics, Faculty of Medicine, The Chinese University of Hong Kong, Hong Kong, China
}

Correspondence should be addressed to Yi-Jian Yang; yyang@cuhk.edu.hk

Received 20 May 2021; Revised 29 June 2021; Accepted 2 September 2021; Published 15 September 2021

Academic Editor: Shuya Chen

Copyright ( 2021 Kin-Chung Wilson Leung et al. This is an open access article distributed under the Creative Commons Attribution License, which permits unrestricted use, distribution, and reproduction in any medium, provided the original work is properly cited.

\begin{abstract}
Most women live with an inactive lifestyle, which suggests a need for preference-based choices to promote their participation in physical activity. This systematic review synthesized key findings on the health benefits of Qigong among women. We conducted a systematic search of randomized controlled trials (RCTs) of Qigong among women according to the PRISMA guidelines using the following databases from their inception through March 2021: PubMed/MEDLINE, Web of Science, Cochrane Library, and US National Library of Medicine. The risk of bias was examined using the Cochrane Collaboration's tool for assessing the risk of bias in randomized trials. Altogether, 18 RCTs were included for final review. Results showed that Qigong was a feasible exercise in improving health outcomes, particularly depressive symptoms (63\% of trials), quality of life (43\%), and fatigue (29\%), among general women, intimate partner violence survivors, and women with chronic conditions (e.g., breast cancer patients or survivors). Almost 90\% (7/8) of trials reported high adherence rates ranging from 73 to $95 \%$ for supervised group training and 63 to $80 \%$ for home self-practice. Thus far, there was no evidence of serious adverse effects from performing Qigong. For the risk of bias across trials, a lack of allocation concealment (72\% of trials), no blinding of participants and personnel (67\%), and incomplete outcome data (67\%) were the major sources. In summary, Qigong is a safe, feasible, and beneficial exercise for general women, abused sufferers, and health-compromised women. However, given the potential risk of bias found in many studies, improved rigor of study design in future trials will be imperatively required.
\end{abstract}

\section{Introduction}

Despite living longer, women generally have poorer health and lower quality of life than men [1]. This is largely due to complexity of biological and gender-related factors such as violence, early marriage, and inequitable access to healthcare [1]. Increasing evidence suggests that women living with a sedentary lifestyle are vulnerable to disabilities, morbidities, and premature death [2]. A 2018 World Health Organization (WHO) report has shown that the global inactivity prevalence in women (27\%) was higher than that in men (20\%) $[3,4]$. To meet the 2025 global target for inactivity (i.e., $10 \%$ reduction) for a healthier world [4], engaging more women in habitual physical activity (PA) to reduce inactivity levels [3] has become a priority in public health.
Several studies have indicated gender differences in PA participation [5-7]. Typically, women are less likely to achieve the WHO-recommended PA levels for health than men throughout the life course [8-11]. From young childhood through late adolescence, a latent class analysis identified three trajectory classes of organized sport participation that were specifically found in boys and girls; "consistent sport participators" for boys (55\%) and girls (48\%) exhibited the best health profile regarding lean body mass, percent body fat, and perceived physical health [12]. The "sport nonparticipator" class was unique to girls (18\%), suggesting that early engagement in organized sport is particularly important in females because they are usually less likely to join sport activities later in life. In middle-aged adults (at the age of 50-65 years), a lower proportion of 
women $(28 \%)$ than men $(41 \%)$ who met the recommended PA guidelines for health was consistently observed [13]. Older women (aged 60 years and older) were also found to participate less in leisure-time PA as measured by both selfreported ( -0.8 to $-21.4 \%)$ and accelerometer-based $(-0.2$ to $-1.5 \%)$ methods [10]. Given that marked gender-specific interests in PA participation persist across the lifespan $[14,15]$, addressing gender preferences for organized activities to enhance PA participation has become a research imperative.

Qigong is a traditional Chinese mindfulness-based exercise characterized by an integration of meditation, breathing, interlacing body movements, and coordinated body posture. "Qi" (pronounced "chee") represents the essential energy flowing inside the human body, while "Gong" (pronounced "gung") refers to regular practice in order to cultivate Qi. Unlike exercise therapies that only target a specific joint or a series of alternate muscle contraction and relaxation, Qigong focuses more on building self-awareness of how the entire body moves through posture and stillness so as to achieve deep states of bodymind relaxation and calmness [16]. Typically, Qigong is of lower impact than endurance training and can be done at any level of exercise tolerance, including accommodation for seated or supine exercises [17-20]. Compared to other mind-body exercises (e.g., Yoga), which share similar principles (meditation, breath focus, and bodily movements), Qigong, a simpler, repetitive form, is believed to be most suitable for women who are less likely to participate in and gain from vigorous activities that require sophisticated skills [15, 21]. For instance, Baduanjin, the most common form of Qigong (56\%) used across 886 primary and secondary research studies [22], is less cognitively demanding than Yoga as Baduanjin is standardized to have eight sections, while Yoga routine is not standardized and has a large variety of movements [23]. Meanwhile, Baduanjin requires a lesser extent of physical demands (e.g., balance, joint stretching, and coordinated body movements) than Yoga $[18,23]$. Other factors that practicing Qigong may fascinate women include relief from emotional distress (e.g., chronic psychological stress and depression [24]), weight loss (e.g., fat-burning [25]), improved appearance (e.g., reduced waist circumference [26]), and friendship building (i.e., group exercise) $[15,27,28]$. Thus far, studies found that more than $90 \%$ of women practitioners were willing to continue practicing Qigong after joining [16, 25, 29], and there were no adverse side effects of Qigong reported, even in critically ill patients [20, 30, 31]. However, owing to a lack of public awareness, qualified instructors, and existing dedicated programs [32], Qigong seems less familiar than other types of mind-body exercises to Westerners [17].

Despite the fact that recent literature reviews have demonstrated potential benefits of Qigong for depressive symptoms [33], body composition [34], and breast cancer [35], which are primary health concerns of women [36-38], a systematic literature review examining women-specific outcomes is still lacking. Besides, most results in recent relevant reviews were obtained from trials varying from randomized controlled trials (RCTs) to quasi-experimental, cohort, and case-control studies [17, 20, 39, 40]. This raises concern over their strength of evidence. A comprehensive review of the benefits of Qigong on women can uncover its potential efficacy on women who have multifaceted needs (e.g., emotional support or humanistic care) that are unsolved by conventional therapies. Therefore, the objectives of this systematic review are twofold: (1) to synthesize evidence obtained from RCTs on the health benefits of Qigong in women categorized by three major conditions (i.e., general women, abused survivors, and women with chronic conditions) and (2) to describe the risk of bias across the included trials. Our findings may provide insightful ideas for practitioners concerning the development of an effective Qigong program that may appeal to different women's motivational profiles.

\section{Materials and Methods}

This systematic review followed the Preferred Reporting Items for Systematic Reviews and Meta-Analyses (PRISMA) guidelines. In line with the PRISMA Checklist [41], the first author developed the study protocol, which was then approved by the coauthors prior to data collection. Reporting of the study flow and findings was in accordance with the PRISMA statement [42].

2.1. Inclusion and Exclusion Criteria. All RCTs examining standalone Qigong as a major treatment among female participants were included. Due to different physiological bases of external $Q i$ therapy that refers to a process by which an experienced Qigong practitioner (i.e., acting as a healer) emits his/her $Q i$ to clear $Q i$ blockage/stagnation, boost $Q i$ levels, or balance $Q i$ activity of another patient, only supervised or self-practice Qigong exercises with meditation and breath focus for enhancing health-related outcomes were considered.

The inclusion criteria were: (1) full text articles, (2) Chinese/English language, (3) peer-reviewed original research, (4) RCT design, and (5) general women, abused survivors, and women with chronic conditions. The exclusion criteria were conference abstracts/reviews, qualitative studies, or studies other than original research such as reviews, meta-analyses, study protocols, comments, letters, case reports, and guidelines.

2.2. Databases and Search Terms. Studies published in the databases of PubMed/MEDLINE, Web of Science, Cochrane Library, and US National Library of Medicine were searched from their inception through March 2021. The text word terms used in the database search (title/abstract/subject/ keywords) included qigong, qi-gong, qi gong, chi chung, chi gong, chi kung, qi chung, Baduanjin, Ba Duan Jin, Liuziju, Liuzijue, Wuqinxin, Wu Qin Xin, Yijinjing, Yijin Jing, Yi Jin Jing, women, woman, and female. Based on the findings of three recent systematic reviews on Qigong and supportive cancer care $[17,20,31]$, two text word terms, namely "breast cancer" and "gynecological cancer", were additionally used for the search. The database search was prerun twice in order 
to refine the text word terms and strategies (e.g., limiters) used in the search. The final search queries for each database with limiters are summarized in Supplementary Materials.

2.3. Article Selection and Review. The eligibility of the searched articles was initially screened based on the title and abstract, followed by the full text. In the initial screening, the searched articles were included and excluded according to the inclusion and exclusion criteria, respectively. Once the information had not been verified in the initial screening, full-text assessment for these studies along with other eligible articles was conducted. To avoid missing any eligible studies, the reference lists of all searched articles or review papers were also screened for additional studies. All duplicates were removed while screening the publications. The data obtained were then summarized into an evidence table, and the coauthors checked and edited each entry for accuracy and consistency. Consensus for relevancy was also made by discussion among the authors.

2.4. Data Collection. Study characteristics of the included studies were summarized using a PICO (P: patient, population, and problem; I: intervention; C: comparison; O: outcome) approach. For each study, data on year of publication, country, participant characteristics (e.g., age, health/clinical status, etc.), sample sizes, intervention conditions (e.g., type, frequency, and duration) and logistic (e.g., supervised training or home practice), attrition rates, outcome measures, and key findings were retrieved and tabulated.

2.5. Risk of Bias Assessment. The risks of bias of the included studies were examined using the Cochrane Collaboration's tool for assessing the risk of bias in randomized trials $[43,44]$. In brief, the tool is composed of seven domains for checking biases: (1) random sequence generation (selection bias), (2) allocation concealment (selection bias), (3) blinding of participants and personnel (performance bias), (4) blinding of outcome assessors (detection bias), (5) incomplete outcome data (attrition bias), (6) selective outcome reporting (reporting bias), and (7) other sources of bias, including the timing of outcome assessment, similarity in randomized groups at baseline, appropriate means for monitoring subject compliance, the appropriate rationale for control groups, fidelity check, sample size estimation, and appropriate judgements for the measurement tools. Studies were classified as having a high risk of bias when one or more bias domains were rated high risk or two or more domains were rated unclear risk. Trials were defined as having a low risk of bias if no bias domain was rated high risk or only one domain was rated unclear risk [45].

\section{Results}

3.1. Study Selection. By March 2021, our database search retrieved 656 records, of which 194 were duplicates. After removing the duplicates, 462 articles were initially screened on their titles and abstracts. The initial screening resulted in 156 articles that were assessed as full text. After the full-text assessment, 138 studies were excluded due to the following reasons: (1) language other than English or Chinese $(n=3)$, (2) no results reported $(n=5)$, (3) non-female-specific participants $(n=109)$, (4) not RCTs $(n=4)$, (5) Qigong combined with other non-Qigong interventions in a multimodal program $(n=2)$, and (6) multiple reasons $(n=15)$. Finally, a total of 18 studies that met the eligibility criteria were included for review (Figure 1).

3.2. Study Characteristics. Characteristics of the 18 included studies are summarized in Table 1. The included studies were published from 1996 to 2020 and conducted in seven places, including China $(n=8)[23,24,26,46-50]$, United States $(n=5)$ [18, 51-54], Australia $(n=1)$ [30], Malaysia $(n=1)$ [55], Spain $(n=1)$ [56], Taiwan $(n=1)$ [57], and Thailand $(n=1)$ [58]. Two studies were published in Chinese [26, 49], and the rest were published in English. The sample sizes ranged from 14 to 271 in total and 8 to 136 in the experimental groups. Of the 18 included studies, 3 (17\%) were conducted in general women (i.e., young [56], middle-aged [57], and menopausal [49]), 1 (6\%) was carried out in abused women [24], and 14 (78\%) were conducted in women with chronic conditions, including breast cancer patients/survivors $(n=8)[30,47,48,50-53,55]$ or patients with diabetes $(n=2)[26,58]$, fibromyalgia $(n=2)[18,54]$, knee osteoarthritis $(n=1)$ [23], and chronic fatigue syndrome $(n=1)$ [46]. Baduanjin ( $n=6)[23,24,26,46,50,57]$ and Liuzijue $(n=3)[18,53,54]$ were the most commonly used Qigong, followed by Guolin Qigong $(n=2)$ [47, 48], Qigong/Tai Chi Easy $(n=2)$ [51, 52], and other Qigong styles $(n=5)$ $[30,49,55,56,58]$. The intervention durations and supervised training frequency varied from 4 to 24 weeks and 1 to 6 times/week, respectively. Control groups that were commonly used across studies included sham exercise (i.e., mimicking Qigong bodily movements without meditation and breath focus; $n=5)[18,51-54]$, no treatment $(n=5)$ $[23,26,50,56,57]$, and usual care $(n=4)[46,47,55,58]$.

3.3. Potential for Bias. All included studies demonstrated some potentials for bias. Figure 2 summarizes the results of the risk of bias analysis for each study. Overall, 94\% (17/18) of the studies had a high or unclear risk of bias in at least one of the seven bias domains [18, 23, 26, 30, 46-58]. Major sources of bias were a lack of allocation concealment (13/18 (72\%)) $[18,23,26,30,46,47,49,51-54,56,57]$, no blinding of participants and personnel (12/18 (67\%)) $[18,23,26,30,46-50,56-58]$, and incomplete outcome data $(12 / 18(67 \%))[18,23,26,30,46,48,49,53-57]$. In addition, only $44 \%(8 / 18)$ of studies clearly described the method of how the random sequence was generated $[24,47,48,50-52,54,55]$. Four studies (22\%) did not specify the numbers and reasons for dropouts [26, 46, 49,57]. Seven studies $(39 \%)$ reported an attrition rate of $20 \%$ or higher $[18,23,30,48,53-55]$, which is considered as the threshold for significant loss of power in validity [59]. Other biases that may render risk of bias to the results included no details 


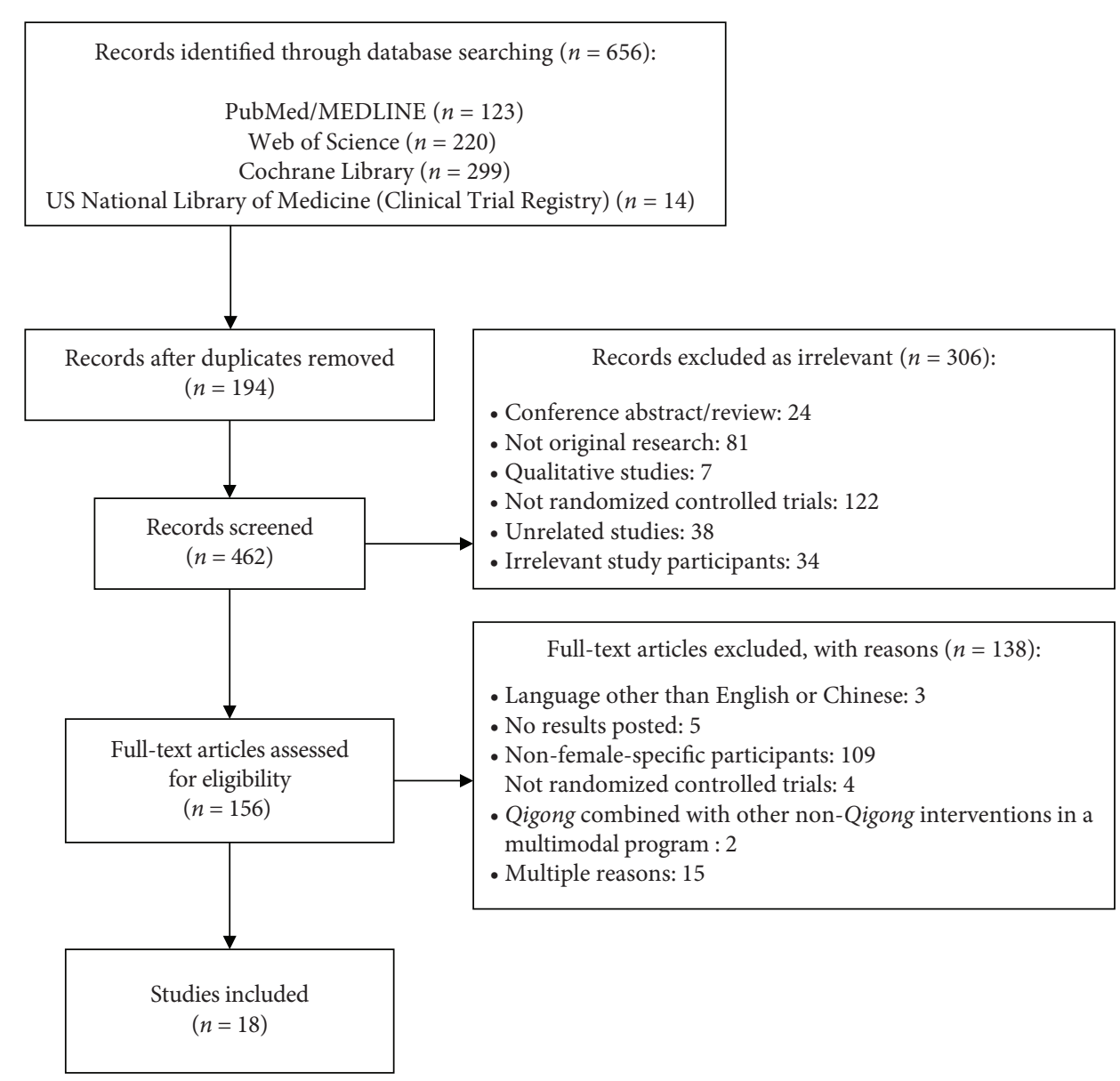

FIgURE 1: Flow chart of the article selection process.

TABLE 1: Characteristics of the included randomized controlled trials published from 1996 to $2020(n=18)$.

\begin{tabular}{lc}
\hline & Number of study (\%) \\
\hline Language & $16(88.9)$ \\
English & $2(11.1)$ \\
Chinese & \\
\hline Place & $8(44.4)$ \\
China & $5(27.8)$ \\
United States & $1(5.6)$ \\
Australia & $1(5.6)$ \\
Malaysia & $1(5.6)$ \\
Spain & $1(5.6)$ \\
Taiwan & $1(5.6)$ \\
Thailand & \\
\hline Study participants & $14(77.8)$ \\
Women with chronic conditions & $3(16.7)$ \\
General women & $1(5.6)$ \\
Abused women & \\
\hline Qigong types & $6(33.3)$ \\
Baduanjin & $3(16.7)$ \\
Liuzijue & $2(11.1)$ \\
Guolin & $2(11.1)$ \\
Qigong/Tai Chi Easy & $5(27.8)$ \\
Others &
\end{tabular}

about monitoring of subject compliance throughout the study $(4 / 18(22 \%))[23,49,56,57]$, no clear description of the rationale for the control groups chosen (12/18 (67\%)) $[23,24,26,30,46,47,49,50,55-58]$, and no priori sample size estimation for the smallest scientifically meaningful effect size $(9 / 18(50 \%))[18,23,26,30,46,49,54,56,57]$.

\section{Health Benefits of Qigong on Female Practitioners}

4.1. General Women. Three trials were conducted in general women (Table 2): (1) young women (aged 18-25 years) [56], (2) middle-aged women (aged 35-60 years) [57], and (3) menopausal women with the climacteric syndrome of durations varying from 4 months to 6 years [49]. The three studies had a high risk of bias (Figure 2).

Wu et al. (1996) [49] showed that climacteric symptoms (e.g., hot flushes, sweating, sleep disturbances, weight gain, mood changes, etc.; $p<0.01$ ) and autonomic functions/ balance $(p<0.05)$ were improved in menopausal women after practicing Qigong versus the drug-treated control group. Serum levels of follicle-stimulating hormone and estradiol were also improved after the intervention $(p<0.05)$. 

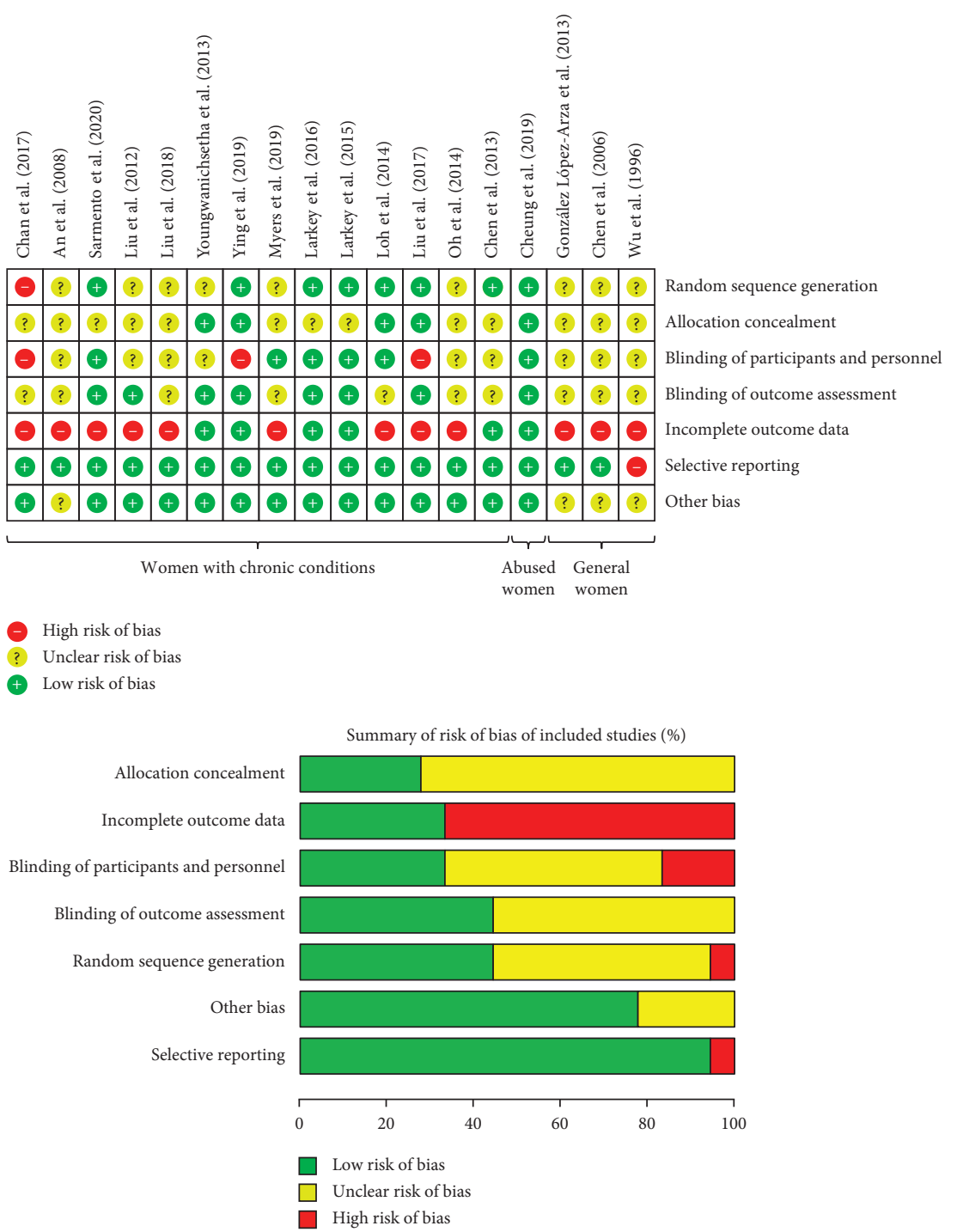

Figure 2: Risk of bias assessment.

TABLe 2: Summary of randomized controlled trials included in the systematic review $(n=18)$.

\begin{tabular}{|c|c|c|c|c|c|c|c|c|}
\hline \multirow[b]{3}{*}{$\begin{array}{l}\text { Author and } \\
\text { place }\end{array}$} & \multicolumn{3}{|c|}{ Study participants } & \multirow{3}{*}{ Intervention } & \multirow{3}{*}{ Control } & \multirow{3}{*}{$\begin{array}{l}\text { Attrition } \\
\text { rate }\end{array}$} & \multirow{3}{*}{ Outcome measures } & \multirow{3}{*}{ Key findings ${ }^{\top}$} \\
\hline & \multirow{2}{*}{$\begin{array}{l}\text { Health } \\
\text { condition }\end{array}$} & \multicolumn{2}{|c|}{ Age (years) } & & & & & \\
\hline & & Range & $\begin{array}{c}\text { Mean/ } \\
\text { median }\end{array}$ & & & & & \\
\hline \multicolumn{9}{|c|}{ General women } \\
\hline $\begin{array}{l}\text { Wu et al. } \\
\text { (1996), China } \\
{[49]^{9}}\end{array}$ & Menopausal & $40-60$ & 50 & $\begin{array}{l}\text { Unspecified } \\
Q G(n=32) ; 1 \\
\text { month }\end{array}$ & $\begin{array}{l}\text { Treated with } \\
\text { vitamins E, } \\
\text { B6, and/or } \\
\text { oryzanol } \\
(\mathrm{n}=18)\end{array}$ & $\begin{array}{c}\text { QG: 6\% } \\
\text { Control: } \\
17 \%\end{array}$ & $\begin{array}{l}\text { a) Climacteric } \\
\text { syndrome (KI) } \\
\text { b) Autonomic } \\
\text { balance (Wengerll) } \\
\text { c) Serum FSH } \\
\text { (RIA) } \\
\text { d) serum LH (RIA) } \\
\text { e) serum } 2 \text { (RIA) } \\
\text { f) serum P (RIA) } \\
\text { g) serum T (RIA) }\end{array}$ & $\begin{array}{l}\text { Within-group } \\
\text { improvement: } \\
\text { (a) } p<0.01 \\
\text { b), d), e) } p<0.05 \\
\text { c), f), g) No } \\
\text { significant changes } \\
Q G / \text { control: } \\
\text { a) } p<0.01 \\
\text { b) } p<0.05\end{array}$ \\
\hline
\end{tabular}


TABle 2: Continued.

\begin{tabular}{|c|c|c|c|c|c|c|c|c|}
\hline \multirow[b]{2}{*}{$\begin{array}{l}\text { Author and } \\
\text { place }\end{array}$} & \multicolumn{3}{|c|}{ Study participants } & \multirow[b]{2}{*}{ Intervention } & \multirow[b]{2}{*}{ Control } & \multirow[b]{2}{*}{$\begin{array}{l}\text { Attrition } \\
\text { rate }\end{array}$} & \multirow[b]{2}{*}{ Outcome measures } & \multirow[b]{2}{*}{ Key findings ${ }^{\top}$} \\
\hline & $\begin{array}{l}\text { Health } \\
\text { condition }\end{array}$ & $\begin{array}{r}\text { Age } \\
\text { Range }\end{array}$ & $\begin{array}{l}\text { (years) } \\
\text { Mean/ } \\
\text { median }\end{array}$ & & & & & \\
\hline $\begin{array}{l}\text { Chen et al. } \\
\text { (2006), Taiwan } \\
\text { [57] }\end{array}$ & Middle-aged & $35-60$ & $\begin{array}{c}\text { QG: } \\
45.7 \pm 6.1 \\
\text { Control: } \\
44.6 \pm 5.5\end{array}$ & $\begin{array}{c}\text { Baduanjin } \\
\text { QG (n=45): } 3 \\
\text { times/week; } \\
12 \text { weeks }\end{array}$ & $\begin{array}{l}\text { No treatment } \\
\qquad(n=45)\end{array}$ & $\begin{array}{c}\text { QG: } 2 \% \\
\text { Control: } \\
4 \%\end{array}$ & $\begin{array}{l}\text { a) Serum IL-6 } \\
\text { (ELISA) } \\
\text { b) BMD } \\
\text { (densitometry) }\end{array}$ & $\begin{array}{l}\text { Within-group } \\
\text { improvement: } \\
\text { a) } p<0.001 \\
\text { b) No significant } \\
\text { changes } \\
Q G / \text { control: } \\
\text { a) } p<0.001 \\
\text { b) } p=0.49\end{array}$ \\
\hline $\begin{array}{l}\text { González } \\
\text { López-Arza } \\
\text { et al. (2013), } \\
\text { Spain [56] }\end{array}$ & Young & $18-25$ & $22.4 \pm 2.5$ & $\begin{array}{c}\text { Wang Ziping } \\
\text { QG }(n=15) ; \\
60 \mathrm{~min} / \\
\text { session; twice } \\
\text { a week; } 4 \\
\text { weeks }\end{array}$ & $\begin{array}{l}\text { No treatment } \\
\quad(n=15)\end{array}$ & $\begin{array}{c}\text { QG: } 13 \% \\
\text { Control: } \\
7 \%\end{array}$ & a) Balance (UBT) & $\begin{array}{l}\text { Within-group } \\
\text { improvement: } \\
\text { a) } p<0.045 \\
Q G / \text { control: } \\
\text { a) No significant } \\
\text { differences }\end{array}$ \\
\hline \multicolumn{9}{|l|}{ Abused women } \\
\hline $\begin{array}{l}\text { Cheung et al. } \\
\text { (2019), China } \\
{[24]}\end{array}$ & $\begin{array}{l}\text { Survivors of } \\
\text { intimate } \\
\text { partner } \\
\text { violence }\end{array}$ & $\geq 18$ & $\begin{array}{c}\text { QG: } \\
42.0 \pm 8.7 \\
\text { Control: } \\
41.5 \pm 9.3\end{array}$ & $\begin{array}{c}\text { Baduanjin } \\
\text { QG (n=136) } \\
\text { Week 1-6 } \\
\text { (training): } \\
120 \text { min/ } \\
\text { session; twice } \\
\text { a week; } 6 \\
\text { weeks } \\
\text { Week 7-22: } \\
60 \text { min/ } \\
\text { session; once } \\
\text { a week; } 16 \\
\text { weeks } \\
\text { Home } \\
\text { practice: } \\
30 \text { min/day; } \\
\text { once daily, 22 } \\
\text { weeks }\end{array}$ & $\begin{array}{l}\text { Wait-list with } \\
\text { health } \\
\text { education } \\
(n=135)\end{array}$ & $\begin{array}{c}\text { QG: } 12 \% \\
\text { Control: } \\
6 \%\end{array}$ & $\begin{array}{l}\text { a) PBMC } \\
\text { telomerase activity } \\
\text { (ELISA) } \\
\text { b) Plasma TNF } \\
\text { (ELISA) } \\
\text { c) Plasma IL-6 } \\
\text { (ELISA) } \\
\text { d) Depression } \\
\text { (BDI) } \\
\text { e) Stress (PSS) } \\
\text { f) Coping (PCS) }\end{array}$ & $\begin{array}{l}\text { Within-group } \\
\text { improvement: } \\
\text { a) } p=0.05 \\
\text { d), e) } p<0.001 \\
Q G / \text { control: } \\
\text { d), e) 6-week: } \\
p=0.009 \text { and } 0.02 \text {, } \\
\text { respectively; } 22- \\
\text { week: No significant } \\
\text { differences } \\
\text { a), b), c), f) No } \\
\text { significant } \\
\text { differences }\end{array}$ \\
\hline \multicolumn{9}{|c|}{ Women with chronic conditions } \\
\hline $\begin{array}{l}\text { Chen et al. } \\
\text { (2013), China } \\
{[47]^{\square}}\end{array}$ & $\begin{array}{l}\text { Breast cancer } \\
\text { patients } \\
\text { receiving } \\
\text { radiotherapy }\end{array}$ & $25-64$ & $\begin{array}{c}\text { QG: } \\
45.3 \pm 6.3 \\
\text { Control: } \\
44.7 \pm 9.7\end{array}$ & $\begin{array}{l}\text { Guolin QG } \\
(n=49) ; \\
40 \mathrm{~min} / \\
\text { session; once } \\
\text { a week; } 5 \\
\text { weeks }\end{array}$ & $\begin{array}{l}\text { Wait-list with } \\
\text { usual care } \\
\quad(n=47)\end{array}$ & $\begin{array}{c}\text { QG: } 2 \% \\
\text { Control: } \\
8 \%\end{array}$ & $\begin{array}{l}\text { a) QOL (FACT-G) } \\
\text { b) Depression } \\
\text { (CES-D) } \\
\text { c) Fatigue (BFI) } \\
\text { d) Sleep quality } \\
\text { (PSQI) } \\
\text { e) Stress/cortisol } \\
\text { rhythm (TRFIA) }\end{array}$ & $\begin{array}{l}\text { Within-group } \\
\text { improvement: } \\
\text { b) } p=0.001 \\
\text { a), c), d), e) No } \\
\text { significant changes } \\
Q G / \text { control: } \\
\text { b) } p=0.05 \\
\text { a), c), d), e) No } \\
\text { significant } \\
\text { differences } \\
\text { Higher baseline } \\
\text { depressive group: } \\
\text { a) } p<0.01 \\
\text { b) } p<0.06 \\
\text { c) } p<0.05 \\
\text { d), e) No significant } \\
\text { differences }\end{array}$ \\
\hline
\end{tabular}


TABle 2: Continued.

\begin{tabular}{|c|c|c|c|c|c|c|c|c|}
\hline \multirow[b]{3}{*}{$\begin{array}{l}\text { Author and } \\
\text { place }\end{array}$} & \multicolumn{3}{|c|}{ Study participants } & \multirow{3}{*}{ Intervention } & \multirow{3}{*}{ Control } & \multirow{3}{*}{$\begin{array}{l}\text { Attrition } \\
\text { rate }\end{array}$} & \multirow{3}{*}{ Outcome measures } & \multirow{3}{*}{ Key findings ${ }^{\top}$} \\
\hline & at & & & & & & & \\
\hline & condition & Range & $\begin{array}{l}\text { Mean/ } \\
\text { median }\end{array}$ & & & & & \\
\hline $\begin{array}{l}\text { Oh et al. } \\
(2014) \text {, } \\
\text { Australia [30] }\end{array}$ & $\begin{array}{c}\text { Metastatic } \\
\text { breast cancer }\end{array}$ & $\geq 18$ & $\begin{array}{c}\text { QG: } \\
56.9 \pm 12.1 \\
\text { Control: } \\
57.8 \pm 10.8\end{array}$ & $\begin{array}{l}\text { Medical QG } \\
(n=14) ; \\
60 \mathrm{~min} / \\
\text { session; once } \\
\text { a week; } 10 \\
\text { weeks } \\
\text { Home } \\
\text { practice: } \\
15-30 \mathrm{~min} / \\
\text { session; } 3-4 \\
\text { times/week; } \\
10 \text { weeks }\end{array}$ & $\begin{array}{l}\text { Meditation } \\
\quad(n=13)\end{array}$ & $\begin{array}{c}\text { QG: } 36 \% \\
\text { Control: } \\
38 \%\end{array}$ & $\begin{array}{l}\text { a) QOL (FACT-B) } \\
\text { b) Fatigue (FACT- } \\
\text { F) } \\
\text { c) Stress (PSS) } \\
\text { d) Neuropathy } \\
\text { (FACT-GOG- } \\
\text { NTX) } \\
\text { e) Sexual function } \\
\text { (SFQ) }\end{array}$ & $\begin{array}{l}\text { QG/control: } \\
\text { d) } p=0.014 \\
\text { a), b), c), e) No } \\
\text { significant } \\
\text { differences }\end{array}$ \\
\hline $\begin{array}{l}\text { Liu et al. } \\
\text { (2017), China } \\
{[48]}\end{array}$ & $\begin{array}{c}\text { Breast cancer } \\
\text { survivors }\end{array}$ & $21-80$ & $\begin{array}{c}Q G: \\
50.9 \pm 7.0 \\
\text { Control: } \\
51.3 \pm 7.3\end{array}$ & $\begin{array}{l}\text { Guolin } Q G \\
(n=79) ; \\
60 \mathrm{~min} / \\
\text { session; twice } \\
\text { a week; } 24 \\
\text { weeks } \\
\text { Home } \\
\text { practice: } 40 \\
\text { min/session; } \\
5 \text { times/week; } \\
24 \text { weeks }\end{array}$ & $\begin{array}{l}\text { Physical } \\
\text { stretching } \\
(n=75)\end{array}$ & $\begin{array}{c}\text { QG: } 41 \% \\
\text { Control: } \\
18 \%\end{array}$ & $\begin{array}{l}\text { a) QOL (FACT-B) } \\
\text { b) Anxiety (HADS) } \\
\text { c) Depression } \\
\text { (HADS) } \\
\text { d) Immunological } \\
\text { markers (serum IL- } \\
2, \text { IFN- } \gamma \text {, and TNF) } \\
\text { (ELISA) } \\
\text { e) Breast cancer- } \\
\text { specific marker } \\
\text { (serum CA 15-3) } \\
\text { (RIA) }\end{array}$ & $\begin{array}{l}\text { Within-group } \\
\text { improvement: } \\
\text { a), c) } p<0.05 \\
\text { b) } p<0.01 \\
\text { d) All markers: } \\
p<0.001 \\
\text { e) } p<0.001 \\
Q G / \text { control: } \\
\text { a) } p=0.002 \\
\text { d) IFN- } \gamma \text { and TNF: } \\
p=0.03 \\
\text { b), c), e) No } \\
\text { significant } \\
\text { differences }\end{array}$ \\
\hline $\begin{array}{l}\text { Loh et al. } \\
\text { (2014), } \\
\text { Malaysia [55] }\end{array}$ & $\begin{array}{c}\text { Breast cancer } \\
\text { survivors }\end{array}$ & $18-65$ & $\mathrm{n} / \mathrm{a}$ & $\begin{array}{l}\text { Zhi Neng QG } \\
\text { (n=66): } \\
90 \mathrm{~min} / \\
\text { session; once } \\
\text { a week; } 8 \\
\text { weeks } \\
\text { Home } \\
\text { practice: } \\
30 \text { min/ } \\
\text { session; twice } \\
\text { a week; } 8 \\
\text { weeks }\end{array}$ & $\begin{array}{l}\text { (i) Line } \\
\text { dancing } \\
(n=65) \\
\text { (ii) Wait-list } \\
\text { with usual } \\
\text { care }(n=66)\end{array}$ & $\begin{array}{c}\text { QG: } 52 \% \\
\text { Line } \\
\text { dancing: } \\
52 \% \\
\text { Usual } \\
\text { care: } 52 \%\end{array}$ & $\begin{array}{l}\text { a) QOL (FACT-B) } \\
\text { b) Fatigue (FACIT- } \\
\text { F) } \\
\text { c) Depression } \\
\text { (DASS-21) } \\
\text { d) Anxiety (DASS- } \\
\text { 21) } \\
\text { e) Stress (DASS-21) }\end{array}$ & $\begin{array}{l}\text { QG/Line dancing: } \\
\text { a) } p=0.036 \\
\text { b), c), d), e) No } \\
\text { significant } \\
\text { differences } \\
Q G / \text { usual care: } \\
\text { a) } p=0.048 \\
\text { b), c), d), e) No } \\
\text { significant } \\
\text { differences }\end{array}$ \\
\hline $\begin{array}{l}\text { Larkey et al. } \\
\text { (2015), United } \\
\text { states [52] }\end{array}$ & $\begin{array}{c}\text { Breast cancer } \\
\text { survivors }\end{array}$ & $40-75$ & $\begin{array}{c}Q G: \\
57.7 \pm 8.9 \\
\text { Control: } \\
59.8 \pm 8.9\end{array}$ & $\begin{array}{c}\text { QG/TCE } \\
(n=49) ; 60 \\
\text { min/session; } \\
\text { 1-2 times/ } \\
\text { week; } 12 \\
\text { weeks } \\
\text { Home } \\
\text { practice: } 30 \\
\text { min/session; } \\
5 \text { times/week; } \\
12 \text { weeks }\end{array}$ & $\begin{array}{c}\text { Sham } Q G^{\ddagger} \\
\quad(n=52)\end{array}$ & $\begin{array}{c}\text { QG: } 14 \% \\
\text { Control: } \\
13 \%\end{array}$ & $\begin{array}{l}\text { a) Fatigue (FSI) } \\
\text { b) Depression } \\
\text { (BDI) } \\
\text { c) Sleep quality } \\
\text { (PSQI) }\end{array}$ & $\begin{array}{l}\text { Within-group } \\
\text { improvement: } \\
\text { b), c) } p<0.05 \\
Q G / \text { control: } \\
\text { a) } p=0.005 \text { at } \\
\text { postintervention; } \\
p=0.024 \text { at } 3 \text {-month } \\
\text { follow-up } \\
\text { b), c) No significant } \\
\text { differences }\end{array}$ \\
\hline
\end{tabular}


TABle 2: Continued.

\begin{tabular}{|c|c|c|c|c|c|c|c|c|}
\hline \multirow[b]{2}{*}{$\begin{array}{l}\text { Author and } \\
\text { place }\end{array}$} & \multicolumn{3}{|c|}{ Study participants } & \multirow[b]{2}{*}{ Intervention } & \multirow[b]{2}{*}{ Control } & \multirow[b]{2}{*}{$\begin{array}{l}\text { Attrition } \\
\text { rate }\end{array}$} & \multirow[b]{2}{*}{ Outcome measures } & \multirow[b]{2}{*}{ Key findings ${ }^{\top}$} \\
\hline & $\begin{array}{l}\text { Health } \\
\text { condition }\end{array}$ & $\begin{array}{r}\text { Ag } \\
\text { Range }\end{array}$ & $\begin{array}{l}\text { (years) } \\
\text { Mean/ } \\
\text { median }\end{array}$ & & & & & \\
\hline $\begin{array}{l}\text { Larkey et al. } \\
\text { (2016), United } \\
\text { States [51] }\end{array}$ & $\begin{array}{c}\text { Breast cancer } \\
\text { survivors }\end{array}$ & $40-75$ & $\begin{array}{c}\text { QG: } \\
57.7 \pm 8.9 \\
\text { Control: } \\
59.8 \pm 8.9\end{array}$ & $\begin{array}{c}Q G / T C E^{\S} \\
(n=49) ; 60 \\
\text { min/session; } \\
\text { 1-2 times/ } \\
\text { week; } 12 \\
\text { weeks } \\
\text { Home } \\
\text { practice: } 30 \\
\text { min/session; } \\
5 \text { times/week; } \\
12 \text { weeks }\end{array}$ & $\begin{array}{l}\text { Sham } Q G^{\ddagger} \\
\quad(n=52)\end{array}$ & $\begin{array}{c}\text { QG: } 14 \% \\
\text { Control: } \\
13 \%\end{array}$ & $\begin{array}{l}\text { a) Mental and } \\
\text { physical QOL (SF- } \\
\text { 36) } \\
\text { b) Cognitive } \\
\text { function (FACT- } \\
\text { COG (subjective) } \\
\text { and WAIS-III } \\
\text { (objective)) } \\
\text { c) PA levels } \\
\text { (BPAQ) } \\
\text { d) BMI }\end{array}$ & $\begin{array}{l}\text { Within-group } \\
\text { improvement: } \\
\text { a) } p<0.001 \\
\text { b) FACT-COG: } \\
p<0.001 \text {; WAIS-III: } \\
p<0.05 \\
\text { c) } p=0.015 \\
\text { d) } p=0.048 \\
\text { Q G/control: } \\
\text { d) } p=0.048 \\
\text { a), b), c) No } \\
\text { significant } \\
\text { differences } \\
\text { 3-month follow-up: } \\
\text { all with no } \\
\text { significant changes } \\
\text { across time or } \\
\text { intergroup } \\
\text { differences }\end{array}$ \\
\hline $\begin{array}{l}\text { Myers et al. } \\
\text { (2019), United } \\
\text { States [53] }\end{array}$ & $\begin{array}{c}\text { Breast cancer } \\
\text { survivors }\end{array}$ & $>18$ & $\begin{array}{c}Q G: \\
52.9 \pm 12.0 \\
\text { Sham } Q G: \\
53.1 \pm 10.7 \\
\text { SS: } \\
56.2 \pm 11.3\end{array}$ & $\begin{array}{l}\text { Liuzijue QG } \\
(n=19) ; \\
60 \mathrm{~min} / \\
\text { session; once } \\
\text { a week; } 8 \\
\text { weeks } \\
\text { Home } \\
\text { practice: } \\
15 \text { min/ } \\
\text { session; twice } \\
\text { daily; } 8 \text { weeks }\end{array}$ & $\begin{array}{l}\text { (i) Sham } Q G^{\neq} \\
(n=20) \\
(\text { ii) } \\
\text { Survivorship } \\
\text { support (SS) } \\
(n=11)\end{array}$ & $\begin{array}{l}\text { QG: } 21 \% \\
\text { Sham } \\
Q G^{\neq}: 50 \% \\
\text { SS: } 0 \%\end{array}$ & $\begin{array}{l}\text { a) Cognitive } \\
\text { function (FACT- } \\
\text { COG (subjective), } \\
\text { RAVLT, and TMT } \\
\text { (objective)) } \\
\text { b) Fatigue } \\
\text { (MDASI) } \\
\text { c) Sleep } \\
\text { disturbance } \\
\text { (MDASI) } \\
\text { d) Distress } \\
\text { (MDASI) } \\
\text { e) PA levels (WHI }\end{array}$ & $\begin{array}{l}\text { QG/sham: } \\
\text { a) TMT: } p=0.007 \\
\text { b), c), d), e) No } \\
\text { significant } \\
\text { differences } \\
Q G / S S: \\
\text { a) FACT-COG: } \\
p<0.05 \\
\text { d) } p<0.02 \\
\text { b), c), e) No } \\
\text { significant } \\
\text { differences }\end{array}$ \\
\hline $\begin{array}{l}\text { Ying et al. } \\
\text { (2019), China } \\
{[50]}\end{array}$ & $\begin{array}{c}\text { Breast cancer } \\
\text { survivors }\end{array}$ & $36-72$ & $54.1 \pm 7.8$ & $\begin{array}{c}\text { Baduanjin } \\
\text { QG }(n=50) \text { : } \\
60 \text { min/ } \\
\text { session; } 3 \\
\text { times/week; } 6 \\
\text { months } \\
\text { Home } \\
\text { practice: } 20 \\
\text { min/session; } \\
4 \text { times/week; } \\
6 \text { months }\end{array}$ & $\begin{array}{l}\text { No treatment } \\
\quad(n=50)\end{array}$ & $\begin{array}{c}\text { QG: } 8 \% \\
\text { Control: } \\
20 \%\end{array}$ & $\begin{array}{l}\text { a) BMI } \\
\text { b) Heart rate } \\
\text { variability (heart } \\
\text { rate monitor) } \\
\text { c) Lung capacity } \\
\text { (spirometry) } \\
\text { d) Arm } \\
\text { circumference on } \\
\text { the affected side } \\
\text { e) Shoulder ROM } \\
\text { on the affected side } \\
\text { (goniometry) } \\
\text { f) } \\
\text { Cardiopulmonary } \\
\text { function (3-min } \\
\text { step test) } \\
\text { g) Anxiety (GAD- } \\
\text { 7) } \\
\text { h) Depression } \\
\text { (PHQ-9) } \\
\text { i) QOL (FACT-B) }\end{array}$ & $\begin{array}{l}\text { QG/control: } \\
\text { b) } p=0.004 \\
\text { e), i) } p=0.000 \\
\text { h) } p=0.020 \\
\text { a), c), d), f), g) No } \\
\text { significant } \\
\text { differences }\end{array}$ \\
\hline
\end{tabular}


TABle 2: Continued.

\begin{tabular}{|c|c|c|c|c|c|c|c|c|}
\hline \multirow[b]{3}{*}{$\begin{array}{l}\text { Author and } \\
\text { place }\end{array}$} & \multicolumn{3}{|c|}{ Study participants } & \multirow{3}{*}{ Intervention } & \multirow{3}{*}{ Control } & \multirow{3}{*}{$\begin{array}{l}\text { Attrition } \\
\text { rate }\end{array}$} & \multirow{3}{*}{ Outcome measures } & \multirow{3}{*}{ Key findings ${ }^{\top}$} \\
\hline & lth & & (years) & & & & & \\
\hline & condition & Range & $\begin{array}{l}\text { Mean/ } \\
\text { median }\end{array}$ & & & & & \\
\hline $\begin{array}{l}\text { Youngwanich } \\
\text { setha et al. } \\
\text { (2013), } \\
\text { Thailand [58] }\end{array}$ & $\begin{array}{l}\text { Diabetic, } \\
\text { postpartum }\end{array}$ & $\mathrm{n} / \mathrm{a}$ & $\begin{array}{c}\text { QG: } \\
35.0 \pm 5.6 \\
\text { Control: } \\
36.2 \pm 4.5\end{array}$ & $\begin{array}{c}\text { Lin Housheng } \\
\text { QG }(n=34) \\
\text { Week } 1 \text { and 2: } \\
50 \text { min/ } \\
\text { session; } 3 \\
\text { times/week; } 2 \\
\text { weeks } \\
\text { Week 3-12: } \\
\text { Home } \\
\text { practice: } 5 \\
\text { times/week; } \\
10 \text { weeks }\end{array}$ & $\begin{array}{l}\text { Usual care } \\
\qquad(n=35)\end{array}$ & $\begin{array}{c}\text { QG: 6\% } \\
\text { Control: } \\
9 \%\end{array}$ & $\begin{array}{l}\text { a) FPG (chemical } \\
\text { analyzer) } \\
\text { b) HbAlc } \\
\text { (chemical analyzer) } \\
\text { c) Blood pressure } \\
\text { d) Body weight, } \\
\text { BMI }\end{array}$ & $\begin{array}{l}Q G / \text { control: } \\
\text { a) } p=0.018 \\
\text { b) } p=0.038 \\
\text { c) Systolic: } \\
p=0.016 \text {; diastolic: } \\
p=0.032 \\
\text { d) No significant } \\
\text { differences }\end{array}$ \\
\hline $\begin{array}{l}\text { Liu et al. } \\
\text { (2018), China } \\
{[26]^{9}}\end{array}$ & $\begin{array}{l}\text { Diabetic, } \\
\text { obese }\end{array}$ & $\mathrm{n} / \mathrm{a}$ & $57.2 \pm 5.4$ & $\begin{array}{c}\text { Baduanjin } \\
\text { QG }(n=20): \\
90 \text { min/ } \\
\text { session; 6 } \\
\text { times/week; } \\
24 \text { weeks (2- } \\
\text { week } \\
\text { training, } \\
\text { followed by } \\
\text { 22-week self- } \\
\text { practice) }\end{array}$ & $\begin{array}{l}\text { No treatment } \\
\quad(n=20)\end{array}$ & $\begin{array}{c}Q G: 15 \% \\
\text { Control: } \\
10 \%\end{array}$ & $\begin{array}{l}\text { a) Body weight, } \\
\text { BMI } \\
\text { b) WC, WHR } \\
\text { c) FPG (chemical } \\
\text { analyzer) } \\
\text { d) HbA1c } \\
\text { (chemical analyzer) } \\
\text { e) TG (chemical } \\
\text { analyzer) } \\
\text { f) TC (chemical } \\
\text { analyzer) } \\
\text { g) LDL (chemical } \\
\text { analyzer) } \\
\text { h) HDL (chemical } \\
\text { analyzer) } \\
\text { i) serum RBP4 } \\
\text { (ELISA) }\end{array}$ & $\begin{array}{l}\text { Within-group } \\
\text { improvement: } \\
\text { b), c), d), e), h), i) } \\
p<0.05 \\
\text { a), f), g) No } \\
\text { significant changes } \\
Q G(\text { control: } \\
\text { b), c), d), e), h), i) } \\
p<0.05 \\
\text { a), f), g) No } \\
\text { significant } \\
\text { differences }\end{array}$ \\
\hline $\begin{array}{l}\text { Liu et al. } \\
\text { (2012), United } \\
\text { States [18] }\end{array}$ & Fibromyalgia & $24-70$ & $\begin{array}{c}\text { QG: } 55.7 \\
\text { Control: } \\
57.5\end{array}$ & $\begin{array}{l}\text { Liuzijue QG } \\
(n=8) ; \\
45-60 \mathrm{~min} / \\
\text { session; once } \\
\text { a week; } 6 \\
\text { weeks } \\
\text { Home } \\
\text { practice: } \\
\text { 15-20 min/ } \\
\text { session; twice } \\
\text { daily; } 6 \text { weeks }\end{array}$ & $\begin{array}{l}\text { Sham } Q G^{\neq} \\
\quad(n=6)\end{array}$ & $\begin{array}{c}\text { QG: } 25 \% \\
\text { Control: } \\
0 \%\end{array}$ & $\begin{array}{l}\text { a) Pain (SMPQ) } \\
\text { b) Fatigue (MFI- } \\
\text { 20) } \\
\text { c) Sleep quality } \\
\text { (PSQI) } \\
\text { d) Fibromyalgia } \\
\text { impact (FIQ) }\end{array}$ & $\begin{array}{l}Q G / \text { control: } \\
\text { a), b), c), d) } \\
p<0.0125\end{array}$ \\
\hline $\begin{array}{l}\text { Sarmento et al. } \\
\text { (2020), United } \\
\text { States [54] }\end{array}$ & Fibromyalgia & $18-70$ & $\begin{array}{c}\text { QG: } \\
42.6 \pm 10.7 \\
\text { Control: } \\
56.1 \pm 12.3\end{array}$ & $\begin{array}{l}\text { Liuzijue QG } \\
\quad(n=14) ; \\
45 \mathrm{~min} / \\
\text { session; once } \\
\text { a week; } 10 \\
\text { weeks } \\
\text { Home } \\
\text { practice: } \\
\text { Twice daily; } \\
10 \text { weeks }\end{array}$ & $\begin{array}{l}\text { Sham } Q G^{\neq} \\
\quad(n=14)\end{array}$ & $\begin{array}{c}\text { QG: } 29 \% \\
\text { Control: } \\
29 \%\end{array}$ & $\begin{array}{l}\text { a) Pain (SMPQ, } \\
\text { VAS, and PPT) } \\
\text { b) Sleep quality } \\
\text { (PSQI) } \\
\text { c) Fatigue (FIQ) } \\
\text { d) Anxiety (HADS) } \\
\text { e) Depression } \\
\text { (HADS) } \\
\text { f) Fibromyalgia } \\
\text { impact (FIQ) } \\
\text { g) QOL (QOLS) }\end{array}$ & $\begin{array}{l}Q G / \text { control: } \\
a), \mathrm{b}), \mathrm{c}), \mathrm{d}), \mathrm{e}), \mathrm{f}) \\
p<0.05 \\
\text { g) } p<0.05 \text { for } \\
\text { control group } \\
\text { presenting greater } \\
\text { improvement }\end{array}$ \\
\hline
\end{tabular}


TABLE 2: Continued.

\begin{tabular}{|c|c|c|c|c|c|c|c|c|}
\hline \multirow[b]{2}{*}{$\begin{array}{l}\text { Author and } \\
\text { place }\end{array}$} & \multicolumn{3}{|c|}{ Study participants } & \multirow[b]{2}{*}{ Intervention } & \multirow[b]{2}{*}{ Control } & \multirow[b]{2}{*}{$\begin{array}{l}\text { Attrition } \\
\text { rate }\end{array}$} & \multirow[b]{2}{*}{ Outcome measures } & \multirow[b]{2}{*}{ Key findings ${ }^{\top}$} \\
\hline & $\begin{array}{l}\text { Health } \\
\text { condition }\end{array}$ & $\begin{array}{r}\text { Age } \\
\text { Range }\end{array}$ & $\begin{array}{l}\text { (years) } \\
\text { Mean/ } \\
\text { median }\end{array}$ & & & & & \\
\hline $\begin{array}{l}\text { An et al. } \\
(2008) \text {, China } \\
{[23]}\end{array}$ & $\begin{array}{c}\text { Knee } \\
\text { osteoarthritis }\end{array}$ & $>55$ & $\begin{array}{c}\text { QG: } \\
65.4 \pm 8.2 \\
\text { Control: } \\
64.6 \pm 6.7\end{array}$ & $\begin{array}{c}\text { Baduanjin } \\
\text { QG }(n=14) \text { : } \\
30 \mathrm{~min} / \\
\text { session; } 5 \\
\text { times/week; } 8 \\
\text { weeks }\end{array}$ & $\begin{array}{l}\text { No treatment } \\
\quad(n=14)\end{array}$ & $\begin{array}{c}\text { QG: } 21 \% \\
\text { Control: } \\
29 \%\end{array}$ & $\begin{array}{l}\text { a) Pain (WOMAC) } \\
\text { b) Stiffness } \\
\text { (WOMAC) } \\
\text { c) Physical } \\
\text { function } \\
\text { (WOMAC) } \\
\text { d) Aerobic capacity } \\
\text { (6-MWT) } \\
\text { e) Peak torque of } \\
\text { the knee extensors } \\
\text { (Isokinetic } \\
\text { dynamometry) } \\
\text { f) General health, } \\
\text { social function, and } \\
\text { mental health (SF- } \\
\text { 36) }\end{array}$ & $\begin{array}{l}\text { QG/control: } \\
\text { a) } p=0.006 \\
\text { b) } p=0.029 \\
\text { c) } p=0.024 \\
\text { d) } p=0.036 \\
\text { e) } p=0.016 \\
\text { f) No significant } \\
\text { differences }\end{array}$ \\
\hline $\begin{array}{l}\text { Chan et al. } \\
\text { (2017), China } \\
{[46]}\end{array}$ & $\begin{array}{l}\text { Chronic } \\
\text { fatigue } \\
\text { syndrome }\end{array}$ & $<50$ & $\begin{array}{c}\text { QG: } 39.5 \\
\text { Control: } \\
42.0\end{array}$ & $\begin{array}{l}\text { Baduanjin } \\
\text { QG }(n=46) \text { : } \\
90 \mathrm{~min} / \\
\text { session; } 16 \\
\text { sessions; } 9 \\
\text { weeks }\end{array}$ & $\begin{array}{l}\text { Wait-list with } \\
\text { usual care } \\
\quad(n=62)\end{array}$ & $\mathrm{n} / \mathrm{a}$ & $\begin{array}{l}\text { a) Plasma } \\
\text { adiponectin } \\
\text { (ELISA) } \\
\text { b) Anxiety (HADS) } \\
\text { c) Depression } \\
\text { (HADS) }\end{array}$ & $\begin{array}{l}\text { QG/control: } \\
\text { a), b) } p<0.05 \\
\text { c) } p<0.001 \\
\text { 3-month follow-up: } \\
\text { all with no } \\
\text { significant } \\
\text { differences }\end{array}$ \\
\hline
\end{tabular}

6-MWT, 6-minute walk test; BDI, Beck Depression Inventory; BMD, bone mineral density; BMI, body mass index; BFI, Brief Fatigue Inventory; BPAQ, Brief Physical Activity Questionnaire; CA 15-3, carcinoma antigen 15-3, CES-D, Center for Epidemiologic Studies Depression Scale; DASS-21, Depression and Anxiety Stress Scale-21; E2, estradiol; ELISA, enzyme-linked immunosorbent assay; FACIT-F, Functional Assessment of Chronic Illness Therapy-Fatigue; FACT-B, Functional Assessment of Cancer Therapy-Breast; FACT-COG, FACT-Cognitive Function; FACT-F, FACT-fatigue; FACT-G, FACT-General; FACT-GOG-NTX, FACT-Gynecologic Oncology Group-Neurotoxicity; FIQ, Fibromyalgia Impact Questionnaire; FPG, fasting plasma glucose; FSH, folliclestimulating hormone; FSI, Fatigue Symptom Inventory; GAD-7, generalized anxiety disorder-7; HADS, Hospital Anxiety and Depression Scale; HbA1c, hemoglobin A1c; HDL, high-density lipoprotein; IFN- $\gamma$, interferon- $\gamma$; IL-2, interleukin-2; IL-6, interleukin-6; KI, Kupperman Index; LDL, low-density lipoprotein; LH, luteinizing hormone; MDASI, MD Anderson Cancer Symptom Inventory; MFI-20, Multidimensional Fatigue Inventory; P, progesterone; PA, physical activity; PBMC, peripheral blood mononuclear cells; PCS, Perceived Coping Scale; PHQ-9, Patient Health Questionnaire; PPT, pressure pain threshold; PSQI, Pittsburgh Sleep Quality Index; PSS, Perceived Stress Scale; QG, Qigong; QOL, quality of life; QOLS, Quality of Life Scale; RAVLT, Rey auditory verbal learning test; SF-36, Short Form-36; SFQ, Sexual Functioning Questionnaire; RBP4, retinol binding protein 4; RIA, ROM, range of motion; radioimmunoassay; SMPQ, short-form McGill Pain Questionnaire; T, testosterone; TC, total cholesterol; TCE, Tai Chi Easy; TG, triglyceride; TMT, trail making test; TNF, tumor necrosis factor; TRFIA, time-resolved fluorescence immunoassay; UBT, unipedal balance test; VAS, visual analog scale; WAIS-III, Wechsler Adult Intelligence Scale-Third Edition; WC, waist circumference; WHI PAQ, women's health initiative brief physical activity questionnaire; WHR; waist-to-hip ratio; and WOMAC, Western Ontario and McMaster Universities Osteoarthritis Index. ${ }^{\circ}$ Only Chinese version is available. ${ }^{\square}$ Participants were randomly assigned to either the $Q G$ or control groups by adaptive randomization, namely "minimization", so the groups were balanced evenly according to demographic information and clinical conditions. ${ }^{\varsigma}$ Tai Chi Easy (QG/TCE) is a simplified form of traditional Tai Chi, which is repeated and also includes $Q G$ bodily movements. The adaptations to the pace, the repetition, and the ease of learning make TCE like a typical $Q G$ exercise. ${ }^{\ddagger}$ Sham $Q G$ mimicked the $Q G$ movements but without healing sounds or meditation and breath focus. "Autonomic response patterns in resting conditions (i.e., salivary output, systolic and diastolic blood pressure, heart period, respiration period, and sublingual temperature) were examined in order to reflect participants' autonomic balance conditions before and after the intervention. ${ }^{\dagger}$ All key findings with significant levels demonstrated improvements in the outcome measures, except specifically indicated. "Within-group improvement" represented within-group improvement for specific outcomes for intervention groups from baseline to the completion of the intervention, while "QG/control", "QG/line dancing", "QG/usual care", "QG/sham", and "QG/SS" represented between-group differences in either percentage or absolute changes from baseline to the completion of the intervention.

Chen et al. (2006) [57] showed that Qigong could delay bone loss $(p<0.049)$ in middle-aged women by reducing serum levels of interleukin-6 $(p<0.001)$ when compared with no treatment control group.

López-Arza et al. (2013) [56] found that Qigong had a significant effect on improving balance $(p<0.45)$ in young women. However, there was no significant difference in the postintervention balance scores between Qigong and no treatment control groups.
4.2. Abused Women. There was one trial conducted in Chinese women who survived intimate partner violence (IPV) in the past two years [24] (Table 2). The primary outcome was telomerase activity, which is a biomarker of women's cellular longevity that is adversely associated with psychological stress [60]. This study had a low risk of bias (Figure 2).

Cheung et al. (2019) [24] showed that telomerase activity was increased in IPV women after practicing Qigong $(p<0.05)$. Ratings of perceived stress and depressive 
symptoms were improved in both intervention $(p<0.001)$ and wait-list control $(p<0.008)$ groups, yet the betweengroup difference was only observed after 6-week training $(p<0.02)$ but not upon the completion of the 22 -week intervention.

\section{Women with Chronic Conditions}

5.1. Breast Cancer Patients/Survivors. Without duplicate counting of participants between Larkey et al. (2015) [52] and Larkey et al. (2016) [51] (i.e., conducted on the same cohort), all trials $(n=8)$ comprised 174 breast cancer patients or survivors (Table 2). Timing of interventions ranged from the early stage in treatment (e.g., receiving active radiotherapy) [47] through the recovery phase after treatment (i.e., survivorship) [48, 50-53, 55]. In one trial, Qigong therapy was studied among women with a confirmed diagnosis of metastatic breast cancer [30]. All studies had a high or unclear risk of bias in at least one of the bias domains, and the major source of bias was a lack of allocation concealment (5/8 (63\%)) [30, 47, 51-53] (Figure 2). Overall, two trials had a low risk of bias $[51,52]$, while the other six studies had a high risk of bias [30, 47, 48, 50, 53, 55].

Health effects of Qigong among breast cancer patients/ survivors were classified according to outcome measures: quality of life (QOL; 6/8 (75\%)) [30, 47, 48, 50, 51, 55], fatigue $(5 / 8(63 \%))[30,47,52,53,55]$, depressive symptoms $(5 / 8(63 \%))[47,48,50,52,55]$, anxiety $(3 / 8(38 \%))$ $[48,50,55]$, sleep $(3 / 8(38 \%))[47,52,53]$, stress $(3 / 8(38 \%))$ $[30,47,55]$, body mass index (BMI; $(2 / 8(25 \%))[50,51]$, cognitive function $(2 / 8(25 \%))$ [51, 53], PA levels $(2 / 8(25 \%))$ $[51,53]$, and other outcomes that were reported once, including distress [53], immunological and breast-cancerspecific markers [48], neuropathy [30], sexual function [30], and physical indicators (e.g., arm circumference, shoulder range of motion, cardiopulmonary function, heart rate variability, and lung capacity) [50]. QOL, fatigue, psychological symptoms (anxiety, depressive symptoms, distress, and stress), and sleep disturbances, which were most frequently reported in studies of Qigong and supportive cancer care $[17,20,31,39,40,61]$, were analyzed as primary outcomes. Other clinical endpoints (e.g., cognitive functions, BMI, PA levels, etc.) were examined as secondary outcomes.

5.1.1. QOL. A self-report instrument, namely the FACT-B (Functional Assessment of Cancer Therapy - Breast; (4/6 $(67 \%))$, was most frequently used to examine breast-cancerspecific QOL [30, 48, 50, 55]. QOL was significantly improved in breast cancer survivors after performing Qigong versus physical stretching $(p=0.002)$ [48], line dancing $(p=0.036)$ [55], usual care $(p=0.048)$ [55], and no treatment $(p=0.000)$ [50]. However, Qigong did not significantly improve QOL in metastatic breast cancer patients [30] or patients receiving radiotherapy [47].

5.1.2. Fatigue. Of the five trials that used different measures for fatigue status, one showed a significant effect of Qigong versus sham exercise in breast cancer survivors $(p=0.005)$ [52]. The other two studies found that Qigong was not effective in improving fatigue in breast cancer survivors when compared with line dancing, usual care, sham exercise, or survivorship support [53, 55]. For metastatic breast cancer patients [30] or patients receiving active treatment [47], there was no significant effect of Qigong versus control groups, such as meditation or usual care.

5.1.3. Psychological Symptoms. For breast cancer survivors, Qigong significantly lowered depression $(p=0.02)[50]$ and distress $(p=0.02)$ scores [53] in two separate studies when compared with no treatment and survivorship support groups, respectively. For breast cancer patients receiving radiotherapy, Qigong was also effective in alleviating depressive symptoms $(p=0.05)$ compared to usual care [47]. However, there was no effect of Qigong on anxiety $[48,50,55]$ and stress $[30,47,55]$.

5.1.4. Sleep. There was no significant effect of Qigong on sleep quality in either breast cancer patients receiving radiotherapy [47] or survivors [52, 53].

5.1.5. Secondary Outcomes. Qigong had a significant effect on improving neuropathy $(p=0.014)$ [30], heart rate variability $(p=0.004)$ [50], shoulder range of motion on the affected side $(p=0.000)$ [50], and immune function ( $p=0.03)$ [48] when compared with the control groups of meditation, no treatment, and physical stretching. However, there was no effect on PA levels [51, 53], sexual function [30], breast-cancer-specific marker (e.g., serum carcinoma antigen 15-3) [48], arm circumference on the affected side [50], cardiopulmonary function [50], and lung capacity [50]. On the other hand, Qigong demonstrated mixed findings on cognitive function $[51,53]$ and $\mathrm{BMI}[50,51]$.

5.2. Diabetic Women. There were two studies conducted in diabetic women: obese [26] and postpartum [58] (Table 2). These two studies had a high risk of bias (Figure 2).

In both obese and postpartum women, Qigong had a significant effect on improving fasting plasma glucose $(p<0.05)$ and hemoglobin A1c levels $(p<0.05)$ when compared with a control group of either no treatment or usual care $[26,58]$. For postpartum women, Qigong was also effective in lowering systolic $(p=0.016)$ and diastolic ( $p=0.032$ ) blood pressure [58]. Moreover, Qigong had an effect on improving waist circumference or waist-to-hip ratio $(p<0.05)$, blood triglyceride $(p<0.05)$, blood highdensity lipoprotein $(p<0.05)$, and serum retinol-binding protein 4 (i.e., a biomarker of glucose metabolism) $(p<0.05)$ in obese diabetic women [26].

5.3. Fibromyalgia Patients. Two trials examined the effects of Liuzijue Qigong on fibromyalgia symptom management in women patients $[18,54]$ (Table 2). These two studies had a high risk of bias (Figure 2). 
Qigong had a significant effect on alleviating fibromyalgia impact $(p<0.05)$, pain $(p<0.05)$, fatigue $(p<0.05)$, sleep disturbances $(p<0.05)$, anxiety $(p<0.05)$, and depression $(p<0.05)$ in fibromyalgia women when compared with sham exercise $[18,54]$. However, patients after practicing sham exercise demonstrated a greater QOL improvement $(p<0.05)$ [54].

5.4. Other Women Patients. Two studies examined the effects of Baduanjin Qigong on knee osteoarthritis [23] and chronic fatigue syndrome [46] (Table 2). These two trials had a high risk of bias (Figure 2).

An et al. (2008) [23] showed that Qigong versus no treatment ameliorated pain $(p<0.006)$, stiffness $(p<0.029)$, physical function $(p<0.024)$, aerobic capacity $(p<0.036)$, and peak torque of the knee extensors $(p<0.016)$ in knee osteoarthritis patients.

Chan et al. (2017) [46] demonstrated that Qigong was more effective than usual care in improving anxiety $(p<0.05)$ and depressive symptoms $(p<0.001)$ through increased levels of plasma adiponectin $(p<0.05)$ in women with chronic fatigue syndrome.

5.5. Adherence to Qigong Programs. Most trials suggested that Qigong programs were well tolerated by the participants. Altogether, 7 out of 8 trials ( $88 \%$ ) reported adherence rates (on a daily or weekly basis) ranging from 73 to $95 \%$ for supervised group training $[18,30,50,52,58]$ and 63 to $80 \%$ for home selfpractice $[18,54,55,58]$. Two studies reported $65-74 \%$ of participants attending $80 \%$ or more of the supervised training sessions [24, 47]. However, one study demonstrated an adherence rate lower than $60 \%$ for both supervised training (52\%) and self-practice (31\%) sessions [53].

5.6. Safety and Adverse Effects. Eight studies indicated that Qigong intervention was safe without adverse side effects among various types of women practitioners $[18,23,24,30,47,51,54,58]$. Although one study reported that four participants had mild knee pain or shoulder problems after practicing Qigong, the symptoms were relieved following action guidance and correction by the instructor [48].

\section{Discussion}

A growing body of studies have examined the potential of mind-body movements to promote health, prevent diseases, and help alleviate disease- or treatment-related symptoms. In the United States, one or more of these mind-body practices comprised up to $30 \%$ of exercise programs in fitness centers and health clubs $[62,63]$, and more women $(10.3 \%$; vs. men $(5.2 \%))$ applied these movements mainly for the purpose of stress reduction [64]. Empirically, the two key components of these movements, breath focus and mind focus, have been proven potentially beneficial for a variety of health outcomes. Slow and focused deep breathing has been found to stabilize blood pressure [65], oxidative stress [66, 67], and autonomic nervous system [65], while meditation has been mostly associated with improved emotional states (e.g., depression) [68-70]. To the best of our knowledge, the present study is the first systematic review synthesizing knowledge concerning potential health benefits of Qigong (a type of mind-body exercise) among women (general women, IPV survivors, and women with chronic conditions), especially in improving depressive symptoms (5/8 (63\%)) [24, 46, 47, 50, 54], QOL (3/ $7(43 \%))[48,50,55]$, and fatigue $(2 / 7(29 \%))[52,54]$.

Depressive disorders [71] and fatigue symptoms [72] are affecting more women than men globally, greatly compromising women's overall QOL. Qigong has been shown to alleviate the severity of psychological symptoms (e.g., depression and anxiety) likely through modulations of the hypothalamic-pituitary-adrenal axis $[73,74]$, monoamine neurotransmitter (e.g., serotonin) [73], brain-derived neurotrophic factor [73], and adiponectin [46]. In particular, breast cancer patients often experience substantial treatment side effects that undermine their daily living; improved QOL and fatigue through regularly practicing Qigong can have a positive impact on their day-to-day life. Our findings showed that Qigong was especially effective in improving QOL and fatigue in breast cancer patients after treatment (i.e., survivorship) $[48,50,52,55]$ but not in women having metastatic cancer [30] or undergoing active radiotherapy [47]. The mixed findings were likely due to persistent psychological distress pertinent to the pretreated disease states, short duration of Qigong program (i.e., 5 weeks [47]), or meditation control group used in the study [30]. In fact, meditation practice has been established as an effective complementary medicine for psychological and behavioural disturbances among breast cancer patients during and after treatment [75-78]. This implicates that the health effects of Qigong were comparable to other evidence-based mindfulness interventions. As opposed to resistance and aerobic exercise in breast cancer patients whose adherence to supervised training was around $70 \%$ and nearly $50 \%$ of them attended $80 \%$ of the sessions [79, 80], the present review reported a relatively higher adherence rate to supervised Qigong programs in breast cancer patients (i.e., 52-95\%) $[30,50,52,53]$, of whom more than $65 \%$ attended at least $80 \%$ of the sessions [47]. This suggests that the tolerance level of supervised Qigong programs by women practitioners was comparable or even superior to aerobic and resistance exercise training, especially in breast cancer patients. Thus, we herein proposed that Qigong is a feasible, preference-based exercise option for women practitioners.

Despite the health benefits of Qigong found in the reviewed studies, improved design in future studies is necessary because many included trials in this review were burdened with a potential risk of bias. The major source was a lack of allocation concealment $(>70 \%)$ and blinding of participants or personnel $(>60 \%)$. Across studies with a low risk of bias in allocation concealment, masking of treatment allocation was most commonly performed using opaque envelope technique $[24,48,50,58]$, where a sequentially numbered, sealed, and opaque envelope informing group assignment was distributed to the participants in order to ensure that the investigators who generated random sequence did not know the group allocation results. To further avoid the disclosure of group allocation, Ying et al. (2019) [50] used 
aluminum foil to make the envelope further invisible, even under intense light. Apart from allocation concealment, blinding of participants from gaining knowledge of Qigong should be done at different levels, including study design (e.g., using sham exercise as control $[18,51-54])$, subject recruitment (e.g., avoiding words "control/sham", "intervention/ experimental", or "Qigong" in the informed consent [54]), and program implementation (e.g., naming of classes using words other than Qigong, such as "rejuvenating movement [51, 52]" or "mild exercise [54]"). However, blinding of participants seems not possible in some scenarios, for example, among patient participants who need to consult family doctors regarding their PA readiness prior to joining exercise programs. To reduce bias for the situation that participant blinding was not feasible, Myers et al. (2019) [53] mentioned that recruitment and consent language should be neutral when describing the intervention and control groups.

To avoid high attrition rates that led to another major source of bias across studies (i.e., incomplete outcome data), Ying et al. (2019) [50] addressed the importance of establishing a rapport with participants in maintaining intervention compliance. Through regular communications by phone, online tools (e.g., WeChat), and face-to-face meetings, participants built a close and warm relationship (like a family) with the researchers involved in the intervention. As a result, only one out of 50 participants withdrew from the intervention due to a vacation trip. Meanwhile, women's PA motivators (e.g., meeting friends, improved appearance, and weight loss) and context preferences (e.g., supervised training, indoor settings, and low cost) should be considered carefully while designing and implementing Qigong programs [15]. To address the most common PA barriers associated with traditional gender roles of women in the family (i.e., lack of time due to caregiving burdens [14, 81, 82]), Qigong allows home practice for practitioners to preserve travelling time for leisure activities as it does not require any equipment or spacious places to be performed at home. Most importantly, Qigong, like Baduanjin, is easy to learn via online videos (https://www. youtube.com/watch?v=UJUNfRyAJww) or other media (e.g., instructional DVDs [30, 47, 48, 51]).

Fidelity of interventions is critically essential as practitioners can optimize health benefits derived from performing correct Qigong movements, and stay away from undesirable side effects of incorrect practice. As an intervention fidelity check, Larkey et al. (2015 and 2016) [51, 52] examined similarity in the level of exertion and perceived equivalency between Qigong and sham exercises (i.e., mimicking Qigong bodily movements without meditation and breath focus) in terms of the presence and strengths of breath focus and meditative connection [62]. Obviously, ratings of "breath focus" and "meditative state" were higher in Qigong versus sham exercise, which shared similar levels of perceived exertion [52]. The frequency and severity of fatigue among breast cancer survivors were significantly ameliorated when compared with sham exercises. On the principle of traditional Chinese medicine, the underlying mechanism of action is the stimulation of blood and $Q i$ circulation, which is a mainstream treatment strategy for chronic fatigue syndrome [83]. Another type of mechanism is the cultivation of a deep sense of relaxation via the focus on breath and meditative states, making Qigong more health advantageous than sham exercises. Long-term Qigong practice is highly recommended as supported by superior effects of long-term practice (vs. short-term) on fatigued breast cancer patients [52, 53, 55] and healthy adults [84]. Regarding safety, as opposed to a systematic review on exercise therapies showing that nearly $40 \%$ of studies reported non-serious adverse events (e.g., pain, fatigue, low back pain, and edema) among participants with or without a medical condition [45], our review showed that only 5\% (4/ 79) of participants in one included study reported recurrence of mild knee pain or shoulder problems from incorrect Qigong movements, but the symptoms were quickly relieved after movement correction [48]. With no evidence of serious adverse events (e.g., death, hospitalization, cerebrovascular accident stroke, and hip fractures), Qigong seems much safer than conventional exercise therapies, even for end-stage disease patients [30]. Taken together, evaluation of intervention fidelity is very important not only to prevent underestimating the relationship between a Qigong intervention and outcome measures but also to avoid drawing false conclusions concerning its effectiveness and safety.

Nonetheless, the findings of this study should be interpreted with caution due to the following limitations. First, a comprehensive meta-analysis was not possibly conducted in the current review as the studies included examined a broad range of outcome measures among a heterogeneity of women practitioners. Second, the validity of our results was challenged by inconsistency in Qigong types examined (Baduanjin, Liuzijue, Guolin, etc.), different types of diseases (e.g., breast cancer, diabetes, fibromyalgia, knee osteoarthritis, or chronic fatigue syndrome), various stages of diseases (e.g., diagnosed with, treated for, and surviving breast cancer), and varying length of interventions. Third, although some studies used the same instrument (i.e., FACT-B) for measuring QOL in breast cancer patients/ survivors [30, 48, 50,55], we were still unable to conclude what dosage of a Qigong intervention (e.g., frequency, intensity, and duration) is most appropriate for improving QOL largely owing to the presence of mixed findings. For instance, when compared with control groups, 6-, 8-, and 24week interventions were effective in improving QOL $[30,48,55]$, yet no significant effect was observed in a 10 week intervention [50]. Factors that largely contributed to the mixed findings were different control group comparisons, various diseases stages (e.g., before and after treatment), different sample sizes, and attrition rates. In view of this, we recommend people to practice Qigong regularly as supported by foreseeable health benefits of long-term practice $[52,53,55,84]$, and no adverse side effects reported thus far. Fourth, diverse control group design across studies would adversely influence the reliability of our findings. Future research adopting a well-designed control group (e.g., sham-controlled interventions) would further uncover the unique health effects of Qigong (e.g., meditative aspects) as opposed to other exercises at similar levels of aerobic exertion. Last but not least, most studies employed subjective measures of health outcomes, which are cognitively reliant 
and hence give rise to substantive recall bias, especially among older adults or cognitively impaired patients.

\section{Conclusion}

Promoting fitness in women is not an easy task, and this underscores a pressing need for preference-based exercise options in order to facilitate their PA participation. Typically, Qigong is of low intensity and easier to adopt than strenuous aerobic or resistance training, thereby favoring women's participation that is likely to be improved with simple exercises in less strenuous formats and without required skills $[15,21]$. This systematic review of RCTs further suggests that Qigong is an evidence-based exercise and potentially beneficial for women, especially on depressive symptoms [24, 46, 47, 50, 54], QOL [48, 50, 55], and fatigue [52, 54]. Compared with aerobic and resistance training, Qigong is more tolerated by women practitioners. This suggests that Qigong is a feasible, preferred activity option for women. Amid the COVID-19 pandemic, many people may suffer from physical inactivity due to hospitalization, bed rest, sustained quarantine, and social distancing. For individuals who want to reduce the COVID-associated sedentariness, self-practicing Qigong at home is evidently feasible with simple forms of movements, high comprehensibility, and no equipment or spacious places required for maintaining physical fitness and health.

\section{Conflicts of Interest}

The authors declare that there are no conflicts of interest.

\section{Acknowledgments}

This work was supported by the scheme of the Knowledge Transfer Project Fund of the Chinese University of Hong Kong (Y.-J.Y, Ref. \#: KPF21GWP34).

\section{Supplementary Materials}

1. PubMed (MEDLINE) search strategy. 2. Web of Science search strategy. 3. Cochrane Library. 4. US National Library of Medicine (Clinical Trial Registry). (Supplementary Materials)

\section{References}

[1] WHO, 10 facts about women's health, WHO, Geneva, Switzerland, 2021, https://www.who.int/features/factfiles/women/ en/.

[2] WHO, Physical Activity and Women, WHO, Geneva, Switzerland, 2021, https://www.who.int/dietphysicalactivity/ factsheet_women/en/.

[3] G. I. Mielke, I. C. M. Da Silva, T. L. Kolbe-Alexander, and W. J. Brown, "Shifting the physical inactivity curve worldwide by closing the gender gap," Sports Medicine, vol. 48, no. 2, pp. 481-489, 2018.

[4] WHO, Reduce physical inactivity, WHO, Geneva, Switzerland, 2021, https://www.who.int/beat-ncds/take-action/policybrief-reduce-physical-inactivity.pdf.
[5] P. C. Hallal, L. B. Andersen, F. C. Bull, R. Guthold, W. Haskell, and U. Ekelund, "Global physical activity levels: surveillance progress, pitfalls, and prospects," The Lancet, vol. 380, no. 9838, pp. 247-257, 2012.

[6] K. Molanorouzi, S. Khoo, and T. Morris, "Motives for adult participation in physical activity: type of activity, age, and gender," BMC Public Health, vol. 15, no. 1, p. 66, 2015.

[7] L.-T. Tsai, F.-E. Lo, C.-C. Yang, J. Keller, and S.-Y. Lyu, "Gender differences in recreational sports participation among Taiwanese adults," International Journal of Environmental Research and Public Health, vol. 12, no. 1, pp. 829-840, 2015.

[8] A. Bauman, F. Bull, T. Chey et al., "The international prevalence study on physical activity: results from 20 countries," International Journal of Behavioral Nutrition and Physical Activity, vol. 6, no. 1, p. 21, 2009.

[9] L. C. Findlay, R. E. Garner, and D. E. Kohen, "Patterns of children's participation in unorganized physical activity," Research Quarterly for Exercise \& Sport, vol. 81, no. 2, pp. 133-142, 2010.

[10] F. Sun, I. J. Norman, and A. E. While, "Physical activity in older people: a systematic review," BMC Public Health, vol. 13, no. 1, p. 449, 2013.

[11] WHO Global, Recommendations on Physical Activity for Health: 65 Years and above, WHO, Geneva, Switzerland, 2011, https://www.who.int/dietphysicalactivity/physical-activity-\% 20recommendations-65years.pdf.

[12] E. K. Howie, J. A. Mcveigh, A. J. Smith, and L. M. Straker, "Organized sport trajectories from childhood to adolescence and health associations," Medicine \& Science in Sports \& Exercise, vol. 48, no. 7, pp. 1331-1339, 2016.

[13] W. Mynarski, "Physical activity of middle-age adults aged 50-65 years in view of health recommendations," European Review of Aging and Physical Activity, vol. 11, no. 141-147, 2014.

[14] C. M. Caperchione, S. Chau, G. J. Walker, W. K. Mummery, and C. Jennings, "Gender-associated perceptions of barriers and motivators to physical activity participation in South Asian Punjabis living in western Canada," Journal of Physical Activity and Health, vol. 12, no. 5, pp. 686-693, 2015.

[15] J. G. Z. Van Uffelen, A. Khan, and N. W. Burton, "Gender differences in physical activity motivators and context preferences: a population-based study in people in their sixties," BMC Public Health, vol. 17, no. 1, p. 624, 2017.

[16] C. Holmberg, J. Rappenecker, J. Karner, and C. M. Witt, “The perspectives of older women with chronic neck pain on perceived effects of qigong and exercise therapy on aging: a qualitative interview study," Clinical Interventions in Aging, vol. 9, pp. 403-410, 2014.

[17] P. J. Klein, R. Schneider, and C. J. Rhoads, "Qigong in cancer care: a systematic review and construct analysis of effective Qigong therapy," Supportive Care in Cancer: Official Journal of the Multinational Association of Supportive Care in Cancer, vol. 24, no. 7, pp. 3209-22, 2016.

[18] W. Liu, L. Zahner, M. Cornell et al., "Benefit of Qigong exercise in patients with fibromyalgia: a pilot study," International Journal of Neuroscience, vol. 122, no. 11, pp. 657-664, 2012.

[19] H. W. Tsang, J. L. Lee, D. W. Au, K. K. Wong, and K. W. Lai, "Developing and testing the effectiveness of a novel health qigong for frail elders in Hong Kong: a preliminary study," Evidence-based Complementary and Alternative Medicine: eCAM, vol. 2013, Article ID 827392, 13 pages, 2013. 
[20] D. Van Vu, A. Molassiotis, S. S. Y. Ching, and T. T. Le, "Effects of Qigong on symptom management in cancer patients: a systematic review," Complementary Therapies in Clinical Practice, vol. 29, pp. 111-121, 2017.

[21] B. Hands, "Male and female differences in health benefits derived from physical activity: implications for exercise prescription," Journal of Women's Health Issues, vol. 5, no. 4, 2016.

[22] Y.-P. Zhang, R.-X. Hu, M. Han et al., "Evidence base of clinical studies on qi gong: a bibliometric analysis," Complementary Therapies in Medicine, vol. 50, Article ID 102392, 2020.

[23] B. An, K. Dai, Z. Zhu et al., "Baduanjin alleviates the symptoms of knee osteoarthritis," Journal of Alternative \& Complementary Medicine, vol. 14, no. 2, pp. 167-174, 2008.

[24] D. S. T. Cheung, W. Deng, S.-W. Tsao et al., "Effect of a qigong intervention on telomerase activity and mental health in Chinese women survivors of intimate partner violence," JAMA Network Open, vol. 2, no. 1, Article ID e186967, 2019.

[25] T. Sakata, Q. Li, M. Tanaka, and F. Tajima, "Positive effects of a qigong and aerobic exercise program on physical health in elderly Japanese women: an exploratory study," Environmental Health and Preventive Medicine, vol. 13, no. 3, pp. 162-168, 2008.

[26] T. Liu, S. Bai, and R. C. Zhang, "Effects of Health Qigong Baduanjin on diabetes related indexes in middle-aged obese women," Zhongguo Ying Yong Sheng Li Xue Za Zhi, vol. 34, no. 1, pp. 19-22, 2018.

[27] M. Kilpatrick, E. Hebert, and J. Bartholomew, "College students' motivation for physical activity: differentiating men's and women's motives for sport participation and exercise," Journal of American College Health, vol. 54, no. 2, pp. 87-94, 2005.

[28] M. R. Weiss and A. L. Smith, "Friendship quality in youth sport: relationship to age, gender, and motivation variables," Journal of Sport \& Exercise Psychology, vol. 24, no. 4, pp. 420-437, 2002.

[29] D. J. Gates and D. Mick, "Qigong," Holistic Nursing Practice, vol. 24, no. 6, pp. 345-354, 2010.

[30] B. Oh, "Effects of qigong on quality of life, fatigue, stress, neuropathy, and sexual function in women with metastatic breast cancer: a feasibility study," Journal of Clinical Oncology, vol. 32, 2014.

[31] P. M. Wayne, M. S. Lee, J. Novakowski et al., "Tai Chi and Qigong for cancer-related symptoms and quality of life: a systematic review and meta-analysis," Journal of Cancer Survivorship, vol. 12, no. 2, pp. 256-267, 2018.

[32] P. Klein, "Qigong in cancer care: theory, evidence-base, and practice," Medicines (Basel, Switzerland), vol. 4, no. 1, 2017.

[33] X. Liu, J. Clark, D. Siskind et al., "A systematic review and meta-analysis of the effects of Qigong and Tai Chi for depressive symptoms," Complementary Therapies in Medicine, vol. 23, no. 4, pp. 516-534, 2015.

[34] L. K. Larkey, D. James, M. Belyea, M. Jeong, and L. L. Smith, "Body composition outcomes of tai chi and qigong practice: a systematic review and meta-analysis of randomized controlled trials," International Journal of Behavioral Medicine, vol. 25, no. 5, pp. 487-501, 2018.

[35] Y. Zeng, X. Xie, and A. S. K. Cheng, "Qigong or tai chi in cancer care: an updated systematic review and meta-analysis," Current Oncology Reports, vol. 21, no. 6, p. 48, 2019.

[36] F. Garawi, K. Devries, N. Thorogood, and R. Uauy, "Global differences between women and men in the prevalence of obesity: is there an association with gender inequality?"
European Journal of Clinical Nutrition, vol. 68, no. 10, pp. 1101-1106, 2014.

[37] WHO International, Agency for Research on Cancer: Cancer Today, WHO, Geneva, Switzerland, 2021, https://gco.iarc.fr/ today/home.

[38] WHO Mental, Health and Substance Use: Gender and Women's Mental Health, WHO, Geneva, Switzerland, 2021, https://www.who.int/teams/mental-health-and-substance-\% 20use/gender-and-women-s-mental-health.

[39] C. L. W. Chan, C.-W. Wang, R. T. H. Ho et al., "A systematic review of the effectiveness of qigong exercise in supportive cancer care," Supportive Care in Cancer, vol. 20, no. 6, pp. 1121-1133, 2012.

[40] M. Soo Lee, K. W. Chen, K. M. Sancier, and E. Ernst, "Qigong for cancer treatment: a systematic review of controlled clinical trials," Acta Oncologica, vol. 46, no. 6, pp. 717-722, 2007.

[41] PRISMA, Key Documents: PRISMA Checklist, PRISMA, Greenville, SC, USA, 2021, http://www.prisma-statement.org/

[42] L. Shamseer, D. Moher, M. Clarke et al., "Preferred reporting items for systematic review and meta-analysis protocols (PRISMA-P) 2015: elaboration and explanation," BMJ, vol. 349, p. g7647, 2015.

[43] J. P. Higgins, “The Cochrane Collaboration's tool for assessing risk of bias in randomized trials," BMJ, vol. 343, no. d5928, 2011.

[44] Collaboration, T. C. The Cochrane Collaboration, Cochrane Handbook for Systematic Reviews of Interventions, 2021, http://www.cochranehandbook.org/.

[45] A. Niemeijer, H. Lund, S. N. Stafne et al., "Adverse events of exercise therapy in randomised controlled trials: a systematic review and meta-analysis," British Journal of Sports Medicine, vol. 54, no. 18, pp. 1073-1080, 2020.

[46] J. S. M. Chan, A. Li, S.-M. Ng et al., "Adiponectin potentially contributes to the antidepressive effects of Baduanjin qigong exercise in women with chronic fatigue syndrome-like illness," Cell Transplantation, vol. 26, no. 3, pp. 493-501, 2017.

[47] Z. Chen, Z. Meng, K. Milbury et al., "Qigong improves quality of life in women undergoing radiotherapy for breast cancer," Cancer, vol. 119, no. 9, pp. 1690-1698, 2013.

[48] P. Liu, J. You, W. T. Y. Loo et al., "Retracted article: the efficacy of Guolin-Qigong on the body-mind health of Chinese women with breast cancer: a randomized controlled trial," Quality of Life Research, vol. 26, no. 9, pp. 2321-2331, 2017.

[49] R. Z. Wu, "Clinical study on QiGong in treating female climacteric syndrome in 50 cases," Shanghai Journal of Traditional Chinese Medicine, vol. 42, no. 2, pp. 26-28, 1996.

[50] W. Ying, Q. W. Min, T. Lei, Z. X. Na, L. Li, and L. Jing, "The health effects of Baduanjin exercise (a type of Qigong exercise) in breast cancer survivors: a randomized, controlled, singleblinded trial," European Journal of Oncology Nursing, vol. 39, pp. 90-97, 2019.

[51] L. K. Larkey, D. J. Roe, L. Smith, and D. Millstine, "Exploratory outcome assessment of Qigong/Tai Chi easy on breast cancer survivors," Complementary Therapies in Medicine, vol. 29, pp. 196-203, 2016.

[52] L. K. Larkey, D. J. Roe, K. L. Weihs et al., "Randomized controlled trial of Qigong/Tai Chi easy on cancer-related fatigue in breast cancer survivors," Annals of Behavioral Medicine, vol. 49, no. 2, pp. 165-176, 2015.

[53] J. S. Myers, M. Mitchell, S. Krigel et al., "Qigong intervention for breast cancer survivors with complaints of decreased 
cognitive function," Supportive Care in Cancer, vol. 27, no. 4, pp. 1395-1403, 2019.

[54] C. V. M. Sarmento, S. Moon, T. Pfeifer et al., "The therapeutic efficacy of Qigong exercise on the main symptoms of fibromyalgia: a pilot randomized clinical trial," Integrative Medicine Research, vol. 9, no. 4, Article ID 100416, 2020.

[55] S. Y. Loh, S. Y. Lee, and L. Murray, "The Kuala Lumpur Qigong trial for women in the cancer survivorship phaseefficacy of a three-arm RCT to improve QOL," Asian Pacific Journal of Cancer Prevention, vol. 15, no. 19, pp. 8127-8134, 2014.

[56] M. V. G. López-Arza, E. Varela-Donoso, J. MontaneroFernández, J. Rodríguez-Mansilla, B. González-Sánchez, and L. González López-Arza, "Qigong improves balance in young women: a pilot study," Journal of Integrative Medicine, vol. 11, no. 4, pp. 241-245, 2013.

[57] H. H. Chen, M. L. Yeh, and F. Y. Lee, "The effects of Baduanjin qigong in the prevention of bone loss for middle-aged women," The American journal of Chinese medicine, vol. 34, no. 5, pp. 741-747, 2006.

[58] S. Youngwanichsetha, S. Phumdoung, and T. Ingkathawornwong, "The effects of tai chi qigong exercise on plasma glucose levels and health status of postpartum Thai women with type 2 diabetes," Focus on Alternative and Complementary Therapies, vol. 18, no. 4, pp. 182-187, 2013.

[59] J. C. Dumville, D. J. Torgerson, and C. E. Hewitt, "Reporting attrition in randomised controlled trials," $B M J$, vol. 332, no. 7547, pp. 969-971, 2006.

[60] E. S. Epel, E. H. Blackburn, J. Lin et al., "Accelerated telomere shortening in response to life stress," Proceedings of the National Academy of Sciences, vol. 101, no. 49, pp. 1731217315, 2004.

[61] Y. Zeng, T. Luo, H. Xie, M. Huang, and A. S. K. Cheng, "Health benefits of qigong or tai chi for cancer patients: a systematic review and meta-analyses," Complementary Therapies in Medicine, vol. 22, no. 1, pp. 173-186, 2014.

[62] L. Larkey, L. Szalacha, C. Rogers, R. Jahnke, and B. Ainsworth, "Measurement pilot study of the meditative movement inventory (MMI)," Journal of Nursing Measurement, vol. 20, no. 3, pp. 230-243, 2012.

[63] L. Larkey, R. Jahnke, J. Etnier, and J. Gonzalez, "Meditative movement as a category of exercise: implications for research," Journal of Physical Activity and Health, vol. 6, no. 2, pp. 230-238, 2009.

[64] D. M. Upchurch and P. J. Johnson, "Gender differences in prevalence, patterns, purposes, and perceived benefits of meditation practices in the United States," Journal of Women's Health, vol. 28, no. 2, pp. 135-142, 2019.

[65] R. Jerath, J. W. Edry, V. A. Barnes, and V. Jerath, "Physiology of long pranayamic breathing: neural respiratory elements may provide a mechanism that explains how slow deep breathing shifts the autonomic nervous system," Medical Hypotheses, vol. 67, no. 3, pp. 566-571, 2006.

[66] S. Bhattacharya, U. S. Pandey, and N. S. Verma, "Improvement in oxidative status with yogic breathing in young healthy males," Indian Journal of Physiology and Pharmacology, vol. 46, no. 3, pp. 349-354, 2002.

[67] D. Martarelli, M. Cocchioni, S. Scuri, and P. Pompei, "Diaphragmatic breathing reduces exercise-induced oxidative stress," Evidence-based Complementary and Alternative Medicine: eCAM, vol. 2011, Article ID 932430, 10 pages, 2011.

[68] P.-S. Chang, M. T. Knobf, B. Oh, and M. Funk, "Physical and psychological effects of Qigong exercise in community- dwelling older adults: an exploratory study," Geriatric Nursing, vol. 39, no. 1, pp. 88-94, 2018.

[69] L. E. Carlson, M. Speca, K. D. Patel, and E. Goodey, "Mindfulness-based stress reduction in relation to quality of life, mood, symptoms of stress and levels of cortisol, dehydroepiandrosterone sulfate (DHEAS) and melatonin in breast and prostate cancer outpatients," Psychoneuroendocrinology, vol. 29, no. 4, pp. 448-474, 2004.

[70] M. B. Ospina, K. Bond, M. Karkhaneh et al., "Meditation practices for health: state of the research," Evidence Report/ technology Assessment, no. 155, pp. 1-263, 2007.

[71] WHO, Depression, WHO, Geneva, Switzerland, 2021, https:// www.who.int/news-\%20room/fact-sheets/detail/depression.

[72] A. Avellaneda Fernandez, "Chronic fatigue syndrome: aetiology, diagnosis and treatment," BMC Psychiatry, vol. 9, p. S1, 2009.

[73] H. W. H. Tsang and K. M. T. Fung, "A review on neurobiological and psychological mechanisms underlying the antidepressive effect of qigong exercise," Journal of Health Psychology, vol. 13, no. 7, pp. 857-863, 2008.

[74] H. W. H. Tsang, W. W. N. Tsang, A. Y. M. Jones et al., "Psycho-physical and neurophysiological effects of qigong on depressed elders with chronic illness," Aging \& Mental Health, vol. 17, no. 3, pp. 336-348, 2013.

[75] R. V. Araujo, A. F. C. Fernandes, I. S. Nery, E. M. L. R. Andrade, L. T. Nogueira, and F. H. C. Azevedo, "Meditation effect on psychological stress level in women with breast cancer: a systematic review," Revista da Escola de Enfermagem da USP, vol. 53, Article ID e03529, 2019.

[76] J. E. Bower, A. D. Crosswell, A. L. Stanton et al., "Mindfulness meditation for younger breast cancer survivors: a randomized controlled trial," Cancer, vol. 121, no. 8, pp. 1231-1240, 2015.

[77] Z. Gok Metin, C. Karadas, N. Izgu, L. Ozdemir, and U. Demirci, "Effects of progressive muscle relaxation and mindfulness meditation on fatigue, coping styles, and quality of life in early breast cancer patients: an assessor blinded, three-arm, randomized controlled trial," European Journal of Oncology Nursing, vol. 42, pp. 116-125, 2019.

[78] H. Greenlee, M. J. DuPont-Reyes, L. G. Balneaves et al., "Clinical practice guidelines on the evidence-based use of integrative therapies during and after breast cancer treatment," CA: A Cancer Journal for Clinicians, vol. 67, no. 3, pp. 194-232, 2017.

[79] K. S. Courneya, R. J. Segal, K. Gelmon et al., "Predictors of adherence to different types and doses of supervised exercise during breast cancer chemotherapy," International Journal of Behavioral Nutrition and Physical Activity, vol. 11, no. 1, p. 85, 2014.

[80] K. S. Courneya, R. J. Segal, K. Gelmon et al., "Predictors of supervised exercise adherence during breast cancer chemotherapy," Medicine \& Science in Sports \& Exercise, vol. 40, no. 6, pp. 1180-1187, 2008.

[81] Y. K. Cheah and B. K. Poh, "The determinants of participation in physical activity in Malaysia," Osong Public Health and Research Perspectives, vol. 5, no. 1, pp. 20-27, 2014.

[82] Y.-J. Chen, Y.-H. Huang, F.-H. Lu et al., "The correlates of leisure time physical activity among an adults population from southern Taiwan," BMC Public Health, vol. 11, no. 1, p. 427, 2011.

[83] J. S. M. Chan, S.-M. Ng, L.-P. Yuen, and C. L. W. Chan, "Qigong exercise for chronic fatigue syndrome," International Review of Neurobiology, vol. 147, pp. 121-153, 2019.

[84] T. Shimazaki, T. Hortobágyi, A. Uematsu et al., "Long-term QiGong practice is associated with improved self-perceived health and quality of life," International Journal of Sport and Exercise Psychology, vol. 18, no. 3, pp. 308-320, 2020. 